\title{
Problemáticas en torno a la gestión de bienes patrimoniales. El caso del patrimonio ferroviario de la localidad de Sundblad (Argentina)
}

\author{
Andrés Pinassi
}

Introducción

El ferrocarril en Argentina ha forjado la identidad de numerosos poblados. Durante los siglos XIX y XX ha contribuido al desarrollo y consolidación de los mismos, sin embargo, en la actualidad se registra una situación totalmente diferente a la de su surgimiento. El cierre de ramales condujo al deterioro de los diferentes complejos ferroviarios y, por ende, al declive de las comunidades. Hoy día, se identifican grandes espacios que funcionan como contenedores de bienes muebles e inmuebles, que en algunos casos propician acciones vandálicas que atentan no solo contra los componentes históricos, sino también contra la propia población local. En este contexto se enmarcan las problemáticas en torno al patrimonio ferroviario de la localidad de Sundblad, emplazada en la provincia de Buenos Aires, que al igual que numerosos pueblos de la llanura pampeana arraigan su historia en el desarrollo del sistema férreo nacional.

El objetivo del trabajo consiste en: analizar la situación actual del conjunto de bienes que estructuran el patrimonio ferroviario del poblado, su relación con los diferentes actores a escala local y con las iniciativas de gestión patrimonial que se vienen desarrollando en pos de la revitalización del sector. Estas últimas acciones forman parte de las tareas de extensión universitaria que en forma particular desarrolla el autor del presente artículo, dado que él configuró gran parte de su espacio vivido en la localidad de Sundblad.

En primera instancia, se presenta el marco conceptual, estructurado con base en los constructos de patrimonio cultural, industrial y ferroviario, adoptados en el trabajo.

Seguidamente, se desarrolla el estudio de caso contextualizado desde una perspectiva geográfica e histórica.

Por último, se analizan los bienes que estructuran el complejo ferroviario local, los actores implicados y las propuestas de gestión desarrolladas.

Con las reflexiones finales se intenta realizar una contribución, al determinar algunos lineamientos que coadyuven a la mejora del patrimonio local y de la calidad de vida de la población. 


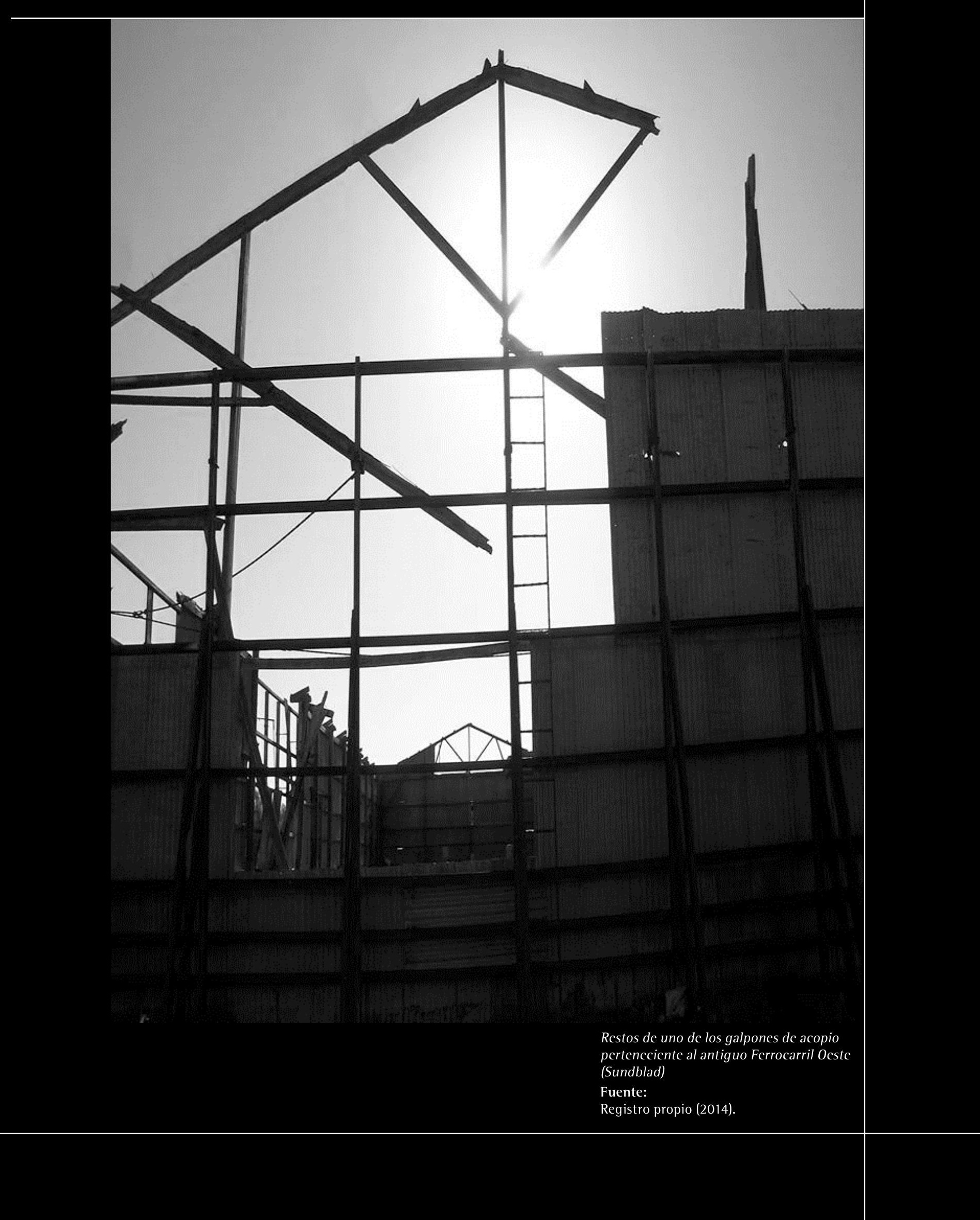




\section{Problemáticas en torno a la gestión de bienes patrimoniales. El caso del patrimonio ferroviario de la localidad de Sundblad (Argentina)}

Issues in the Management of the Cultural Heritage. The Case of the Railway Heritage of Sundblad (Argentina)

Questões relacionadas com a gestão do património cultural. 0 caso

El presente artículo se enmarca en el PGI (Proyecto de Grupos de Investigación):

"Turismo y dinámicas territoriales: diferentes perspectivas de análisis" (24/G076), que se lleva a cabo en el Departamento de Geografía y Turismo de la Universidad Nacional del Sur (Argentina) y es financiado por la Secretaría

General de Ciencia y Tecnología de la misma institución

Agradecimientos

El autor del presente artículo quiere manifestar su agradecimiento a Gloria Mayoral, Directora de la Escuela Primaria № 12 , por la información brindada, y a Antonela Pinassi por la colaboración en el relevamiento fotográfico.

Artículos de investigación

Recepción: 21 de noviembre de 2013

Aceptación: 18 de mayo de 2014

Disponible en línea: 10 de diciembre de 2015

do património ferroviário da vila de Sundblad (Argentina)

\section{Andrés Pinassi}

andres.pinassi@uns.edu.ar

Universidad Nacional del Sur, Consejo Nacional de Investigaciones Científicas y Técnicas de la República Argentina. Doctorando en Geografía (Universidad Nacional del Sur). Magíster en Gestión del Patrimonio Arquitectónico y Urbano (Universidad Nacional de Mar del Plata). Licenciado en Turismo (Universidad Nacional del Sur).

\section{Resumen}

La localidad de Sundblad, pequeño poblado de la provincia de Buenos Aires (Argentina), surge asociada al ferrocarril, al igual que la gran parte de las aglomeraciones del país. En la actualidad, resultado de disímiles procesos económicos y sociales, se evidencia un deterioro de su patrimonio ferroviario. En este contexto, el presente artículo analiza las problemáticas en torno a dichos bienes patrimoniales, su gestión y los actores clave implicados. Como aporte, a modo de reflexión, se presentan determinados lineamientos que contribuyen a la puesta en valor del sector a escala local.

Palabras clave: patrimonio cultural; patrimonio industrial; patrimonio ferroviario; Sundblad; Ferrocarril Oeste

Abstract

Sundblad, is a small town in the province of Buenos Aires (Argentina), associated to railroad comes as much from the crowded country. Today, the result of disparate economic and social processes, a deterioration of its railway heritage is evident. In this context, this paper analyzes the issues around these heritage assets, its management and key stakeholders. As a contribution, by way of reflection, certain guidelines are presented, contributing to the enhancement of the sector locally.

Keywords: cultural heritage; industrial heritage; heritage railway; Sundblad; Western Railway

\section{Resumo}

Sundblad, é uma pequena povoada na província de Buenos Aires (Argentina), emerge associado com a estrada de ferro como muito do país. Hoje, resultado de processos econômicos e sociais diferentes, é evidente uma deterioração do seu património ferroviário. Neste contexto, o presente artigo analisa as questões em torno destes bens patrimoniais, a sua gestão e as principais partes interessadas. Como contribuição, a título de reflexão, algumas orientações são apresentados, contribuindo para a melhoria do setor.

Palavras-chave: patrimônio cultural; patrimônio industrial; patrimônio ferroviário; Sundblad; Ferrocarril Oeste 
Definiendo conceptos: patrimonio cultural, industrial y ferroviario

La conceptualización del patrimonio cultural puede abordarse desde diferentes perspectivas: una etimológica, una antropológica y una integral. Desde el primer enfoque, el etimológico, el patrimonio cultural se afianza sobre la base del propio concepto de patrimonio, asociado este a la herencia, propiedad y pertenencia de ciertos objetos. En un sentido colectivo, el patrimonio cultural se configura entonces por el legado social que representa parte de la cultura e identidad de un determinado pueblo. En este contexto, adquiere importancia el concepto desarrollado por la Unesco (1972) en la "Convención sobre la protección del patrimonio mundial, cultural y natural”, el cual considera que en este forman parte los monumentos, conjuntosy lugares que guardan un valor universal excepcional. Esta concepción coloca el énfasis en los objetos, es decir, en el conjunto de bienes traspasados de generación en generación, que deben ser preservados por ciertos valores que albergan. En esta línea se destacan diferentes autores (lcomos, 1999; Hernández, 2002; Crespí y Planells, 2003; Ballart y Tresserras, 2007; Camarero y Garrido, 2008; Pardo, 2008; Costa, 2009; Velasco, 2009; Querol, 2010; Mejía y Valladares, 2011; Acebo y Schlüter, 2012; Arias, 2012; Moreira y Tréllez, 2013; Torrejón, 2013) que hacen un tratamiento centrado en los componentes como "viajeros pasivos" en el tiempo. Es decir, que los elementos son visualizados como recursos que se transfieren entre diferentes generaciones de forma inmutable. No se hace referencia alguna a los cambios producidos a lo largo de la dimensión temporal y a los procesos de valorización social que los mismos tienen durante su vida útil. Cabe plantearse entonces: ¿Qué sucede con los sujetos que aprecian dichos recursos culturales? ¿Por qué prevalece el pasado como dimensión temporal? ¿Qué papel adquiere el presente y el futuro?

Aquí entra en juego el segundo abordaje en la conceptualización del patrimonio: la perspectiva antropológica. Desde ésta se define al patrimonio cultural como una construcción sociocultural, es decir, que el patrimonio se visualiza como algo creado por el hombre (más allá de una obra material o manifestación en sí misma), con un fin específico en un tiempo y espacio dados. En este enfoque se destaca Prats (1997, 1998, $2005,2006,2014)$ quien manifiesta que tal cons- trucción cultural aparece asociada a los discursos o mensajes que un determinado poder imperante (político) quiere comunicar (de forma implícita o explícita) mediante la patrimonialización de ciertos bienes o expresiones. En este contexto cobra protagonismo la dimensión temporal presente, ya que es en el "hoy" cuando se define, piensa y gestiona el propio patrimonio. Asimismo, los sujetos adquieren un rol preponderante, ya que es el hombre ("representado" por un determinado poder) quien determina dicho legado.

Por último, cabe destacar la visión integral. En esta esfera se articulan las dos perspectivas anteriores, es decir, que el patrimonio es visto como un todo complejo en el que se integran bienes y manifestaciones culturales, los hombres en sociedad, la valorización social que ellos realizan sobre un el cúmulo de componentes, el espacio (material y subjetivo) en el que se encuentran inmersos y las relaciones de poder en torno a ellos. En este enfoque podrían incluirse los aportes de Gutiérrez (2014), Zingoni (2014) y García y De Medeiros (2014), quienes manifiestan que el patrimonio cultural no son solo los objetos/manifestaciones, sino el vínculo que los unen a los sujetos, promotores del reconocimiento y apreciación.

En este mismo contexto se destaca la noción de patrimonio territorial (Ortega, 1998; Conti, 2010; Feria, 2010, 2013), la cual excede al patrimonio cultural (material e inmaterial) en forma sesgada, para colocarlo en relación con aquel natural que se desarrolla en un espacio geográfico dado.

Como integrante del patrimonio cultural se evidencia el patrimonio industrial. De acuerdo con lo manifestado por Rodrigues (2010), dicho concepto surge a finales del siglo XIX en Portugal y se populariza en los años cincuenta del siglo $X X$ vinculado con la "arqueología industrial". En este contexto, el TICClH (Comité Internacional para la Conservación del Patrimonio Industrial), expresa que:

El patrimonio industrial se compone de los restos de la cultura industrial que poseen un valor histórico, tecnológico, social, arquitectónico o científico. Estos restos consisten en edificios y maquinaria, talleres, molinos y fábricas, minas y sitios para procesar y refinar, almacenes y depósitos, lugares donde se genera, se transmite y se usa energía, medios de transporte y toda 
su infraestructura, así como los sitios donde se desarrollan las actividades sociales relacionadas con la industria, tales como la vivienda, el culto religioso o la educación (TICCIH, 2003, p. 1).

Este patrimonio se configura, entonces, por el legado de determinadas actividades productivas o económicas de cierta relevancia para un territorio, y que en la actualidad se encuentren o no en funcionamiento, su herencia (bienes materiales y manifestaciones de la cultura) deja sus marcas en el paisaje y, asimismo, determina ciertos modos de vida en torno a dicha actividad.

Como categoría o tipología del patrimonio industrial se destaca el patrimonio ferroviario, el cual está conformado por el conjunto de bienes muebles e inmuebles que constituyeron parte del desarrollo del transporte por tren y sus actividades complementarias. Entre ellos se identifican las estaciones de pasajeros (de paso y de final de recorrido), los galpones de acopio, las propias vías y durmientes, la señalética, los cambios de vía, los tanques y molinos para abastecimiento de la locomotora, entre otros. Este conglomerado de componentes constituye un sistema en relación con otros subsistemas, que funcionan en forma articulada en pos del desarrollo armónico de la actividad (Ferrari, 2010b). Cabe adicionar las manifestaciones, relatos y vivencias que se sucedieron o se dan en torno a este y que hoy en día constituyen parte del patrimonio intangible. En este sentido, el desarrollo del ferrocarril comprende un conjunto de recursos materiales e inmateriales que caracterizan determinados espacios (urbanos o rurales) y que configuran parte de la identidad cultural comunitaria.

En Argentina, el surgimiento de este patrimonio sienta sus bases en la Constitución Nacional de 1853, a partir de una clara visión de expansión territorial por parte del Estado (Ferrari, 2010a). En ese mismo año se conforma la Sociedad del Camino de Hierro de Buenos Aires al Oeste, promotora del Ferrocarril Oeste. En 1854, se presentan los planos para construir la primera estación en la ciudad de Buenos Aires. Finalmente, en 1857, se da apertura al primer tramo ferroviario del país, con una extensión de 10 kilómetros, que se desarrollaba desde la Estación Parque hasta Floresta (Tartarini, 2005). El período de mayor expansión se da de la mano del Modelo Agroexportador. Dicho proceso económico lle- va a la consolidación de la infraestructura y el equipamiento ferroviario en diferentes partes del territorio, dando lugar a numerosos poblados o incentivando el crecimiento de otros. $\mathrm{Al}$ respecto Torres Cano (2008, p. 43) manifiesta:

Todas las crónicas reflejan lo festivo que fue, la llegada del tren y la estación, y como varió el modo de vida, el ritmo de los pueblos, y por último el modo de viajar. [...] El tren cambió radicalmente estas experiencias, transformándolas en una forma de cultura, una práctica social apreciada, deseable, prestigiosa y un placer en sí misma.

A partir de la década de 1930, con la crisis mundial, comienzan a darse los primeros síntomas de decadencia del sistema. Determinados devenires económicos y sociales a escala mundial y nacional comienzan a impactar en el desarrollo de la actividad agrícola que afectan la ferroviaria y la llevan a un déficit que provoca el cierre de ramales. A principios de la década de los años noventa dejan de operar otros recorridos, lo que reduce la actividad de transporte y produce el despido de personal. En este periodo se da la privatización del ferrocarril que conduce a la intensificación del estado de deterioro del sistema (Schvarzer, 1999). Estos devenires determinaron la perspectiva actual de los paisajes de gran parte de los poblados de la Argentina: un conjunto de bienes en desuso y en estado de deterioro que reflejan la historia de una nación próspera. Legado que hoy en día adquiere otra connotación, se transforma en una oportunidad y en un campo de acción propicio para la gestión del patrimonio cultural, disciplina promotora de la revitalización de dichos recursos a partir de su puesta en valor y de la generación de espacios de uso e intercambio comunitario, destinados a la población local o en ciertos casos a visitantes.

\section{Estudio de caso: localidad de Sundblad}

Sundblad es una pequeña localidad de apenas 63 habitantes (Instituto Nacional de Estadística y Censos, 2010), localizada en el noroeste de la provincia de Buenos Aires, en el partido de Rivadavia (Figura 1). Su base productiva se encuentra arraigada en la actividad agrícola ganadera y la apicultura. 
La trama del poblado se conforma por ocho cuadrículas, a las que se adiciona el terreno perteneciente al ferrocarril. Algunas de ellas presentan viviendas en forma dispersa, mientras que otras solo albergan una o dos casas.

Sundblad, al igual que gran parte de las pequeñas localidades de la provincia, surge vinculada al Ferrocarril Oeste'1. El 19 de diciembre de 1911 fue inaugurada la Estación de Tren con el mismo nombre que el pueblo. En este período y durante los años posteriores comienza su estructuración y desarrollo.

La llegada del primer tren, el 31 de diciembre de 1911, condujo a la localidad a albergar a los primeros habitantes que arribaron junto a la empresa. Las vías y la primera vivienda fueron construidas entre 1901 y 1902.

Entre los primeros edificios de la localidad se destacan el almacén, perteneciente al Sr. Antonio Velázquez, y la farmacia, del Sr. Varrenti.

En la década de 1940, el total de habitantes no alcanzaba a superar los setecientos. A cargo de la estafeta postal se hallaba Mariano Fortuna y el hotel era propiedad de Florentino Fraga. Había tres almacenes y un bar, de la firma Marote Hermanos.

La primera escuela fue la $\mathrm{N}^{\circ} 12$, Julio A. Roca, creada el 21 de abril de 1928, con motivo del aumento poblacional de Sundblad. El 3 de marzo de 1942 se rubricó el primer libro de actas de la Asociación Cooperadora, cuando el director a cargo del establecimiento era el Sr. Félix Pedernera y la docente Srita. Elba Bocaccio. Fue construida en un terreno donado generosamente por la familia Velázquez (CITAB, 2004, p. 10).

El Club Juventud Unida, institución deportiva de ese entonces y de la que hoy solo se cuenta con el edificio (refaccionado entre 2013 y 2014), abrió sus puertas el 31 de agosto de 1941.

Las rutinas del transporte ferroviario por esos días eran:

[...] llegaban hasta tres trenes de pasajeros por día, con destino a Once (Capital Federal y Gran Buenos Aires), Winifreda (La Pampa) y dos trenes mixtos dos veces al día con salida a General Pico (La Pampa) y a Trenque Lauquen (Provincia de Buenos Aires).

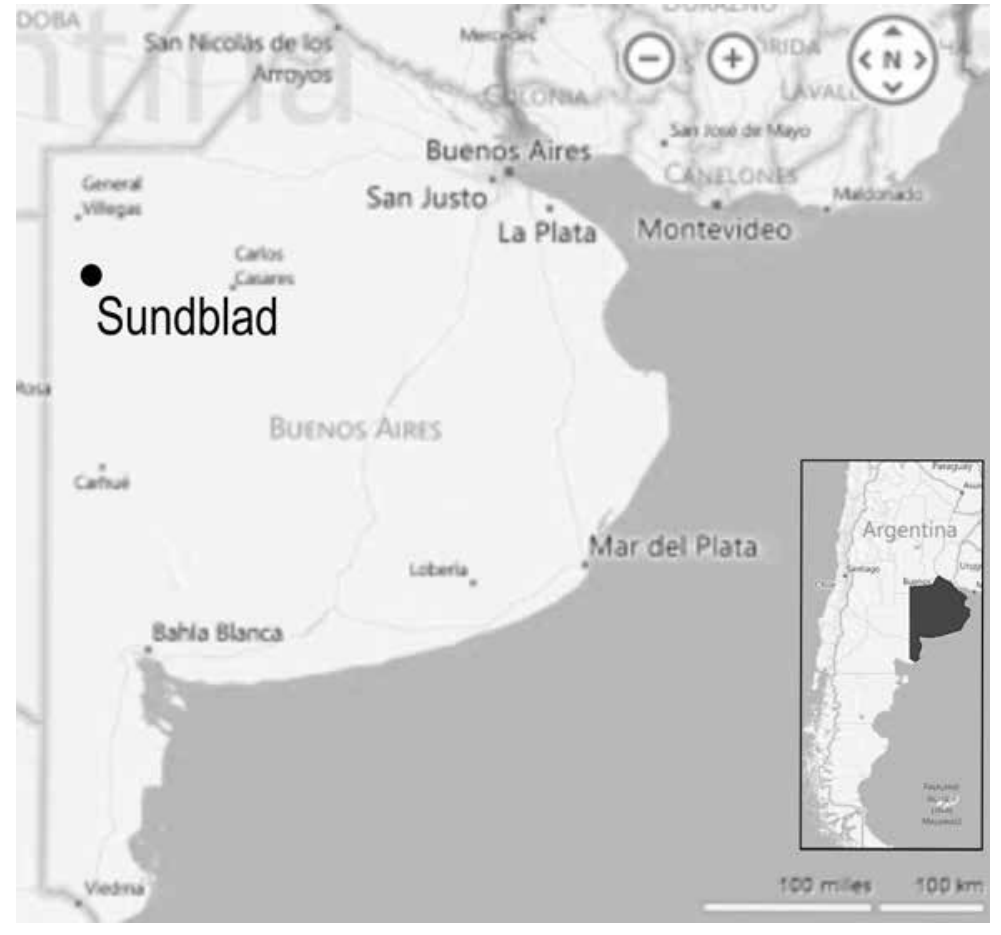

[...] los lunes arribaban trenes de hacienda con destino a América, directo hacia la ciudad de Buenos Aires (Municipalidad de Rivadavia, 2014).

Este período se constituye como el de mayor desarrollo y esplendor de Sundblad. Posteriormente, las sucesivas crisis mundiales impactaron de manera negativa la situación local, en particular la caída del modelo agroexportador, vigente en el país hasta la década de los años cuarenta.

Otros acontecimientos también cambiaron la realidad del territorio: el desalojo de campos, producido en los años 1935 y 1936, la venta de los mismos y la instalación de grandes explotaciones ganaderas. En la década de los años ochenta, más exactamente en los años 1985 y 1986, y de manera reincidente en 2002, se produjeron grandes inundaciones en el partido que provocaron pérdidas irreparables en viviendas y campos. Este cúmulo de factores derivó en un estancamiento económico de la localidad y el área de influencia, junto a la pérdida notoria de habitantes.

\section{Problemáticas y gestión en torno al patrimonio ferroviario local}

El complejo ferroviario se localiza hacia el este del poblado ${ }^{2}$ y está conformado por un conjunto de obras arquitectónicas, entre las que se destacan: la estación de tren, las antiguas viviendas del

Figura 1:

Localización de Sundblad Fuente:

Elaboración propia sobre la base de

Bing Maps (2014)$$
\text { Bing Maps (2014) }
$$

1. Reseña histórica confeccionada a partir de: Ramírez (1997); Centro de Investigaciones Territoriales y Ambientales Bonaerenses (CITAB, 2004a); Pinassi (2012) y Municipalidad de Rivadavia (2014).

2. Catastralmente corresponde a la Circunscripción VII, Sección Rural, Parcela 454. 


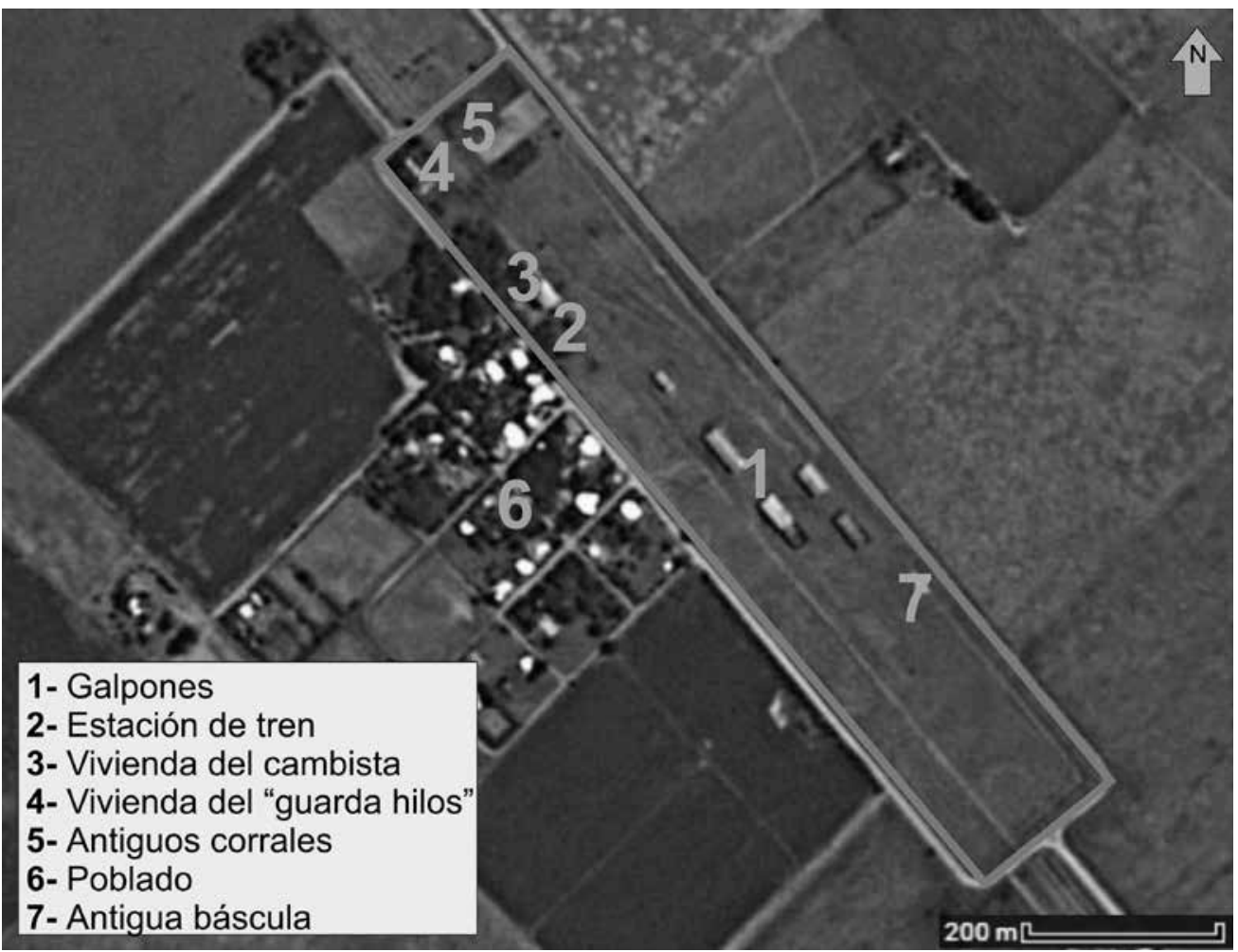

Croquis del complejo

ferroviario

Fuente:

Elaboración propia con base en Google Earth (2014)

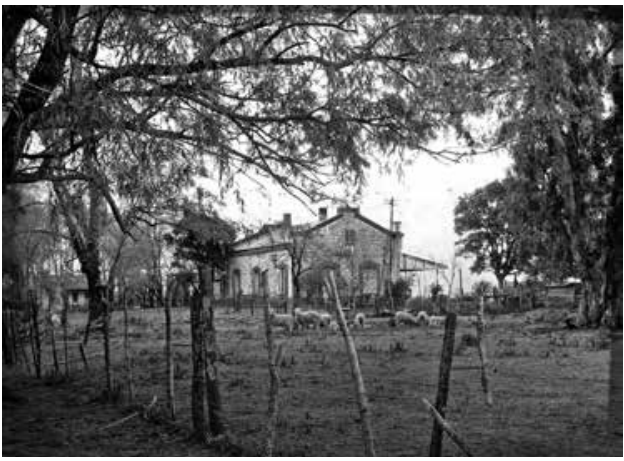

cambista o "ayudante de vía” y del "guarda hilos" o de la persona encargada del mantenimiento de la infraestructura de comunicaciones, un sector de corrales destinado antiguamente al encierro de ganado, las ruinas de una báscula y los galpones de acopio (Figura 2). Además de ello, se puede identificar determinado equipamiento ferroviario que aún se conserva en el sector, parte del cual es un tanque para abastecimiento de agua a la

Figuras 3 y 4 : Estación de tren

Fuente: Registro propio (2014)

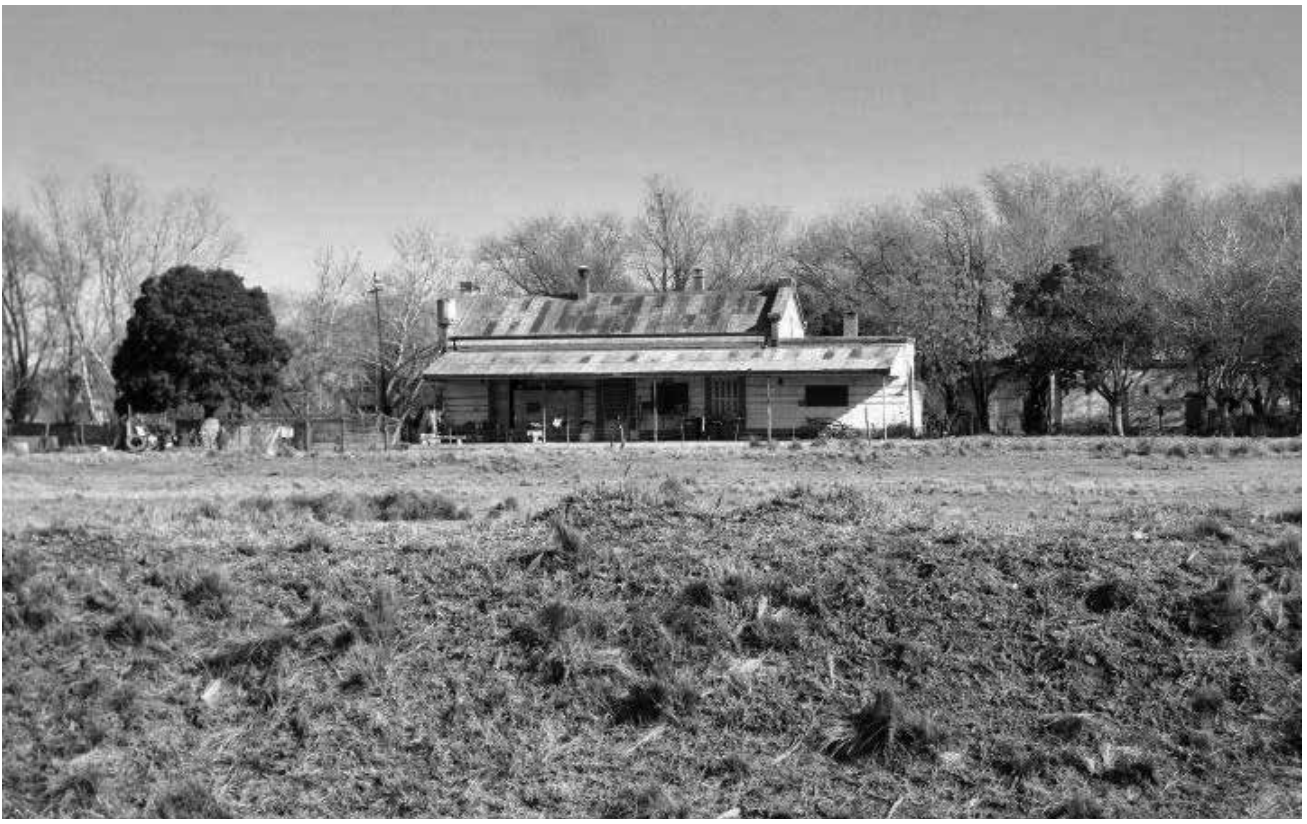



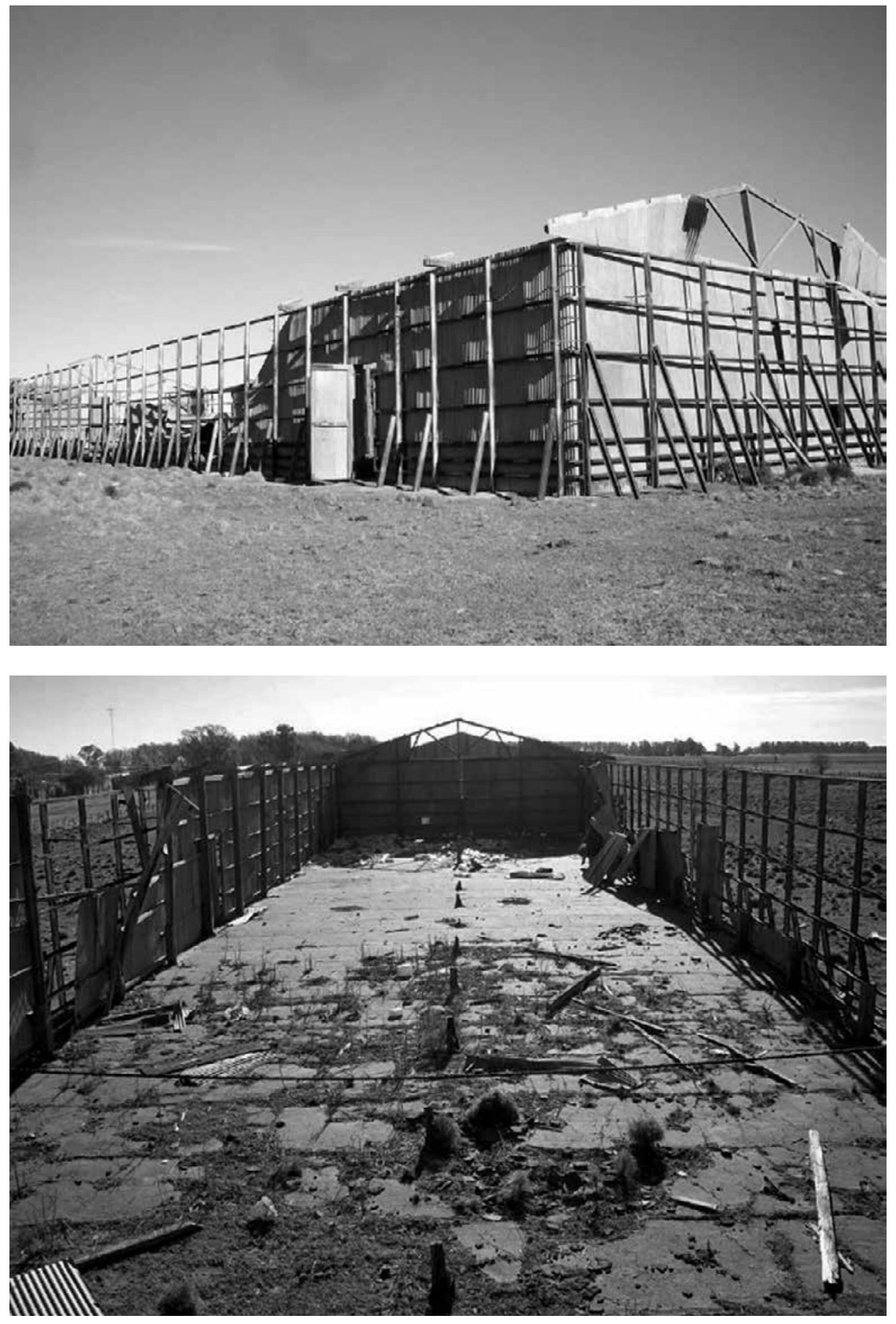

Figuras 5 y 6 : Antiguo galpón de acopio

Fuente:

Registro propio (2014)

locomotora, algunos carteles de señalización, los cambios de vía y los propios rieles.

En términos generales el estado de conservación es de carácter regular, y se torna inadecuado en el caso de algunos de los galpones. La estación y la antigua vivienda del cambista son las mejores preservadas debido a que tienen un uso residencial. En la primera construcción, el edificio se ha dividido en dos dando lugar al alojo de dos familias; para ello, en la zona del andén se ha construido un sanitario para adecuar la funcionalidad de la obra a las necesidades de los moradores. Cabe aclarar que esa intervención no ha sido realizada siguiendo los criterios preservacionistas, bregando por los valores formales del inmueble, sino, por el contrario, se ha erigido una estructura sin cuidado 
alguno (Figuras 3 y 4). La segunda obra también alberga un uso residencial. Si bien la estructura edilicia no ha sufrido alteraciones, ha sido pintada sin respetar los colores originales. En ambos casos, si bien se evidencian deterioros, se manifiesta cierto cuidado por parte de sus resientes.

En relación con el antiguo edificio del "guarda hilos”, la antigua casona se encuentra inutilizada, por lo que denota un estado de deterioro y carente de mantenimiento.

Estos tres bienes se destacan por su arquitectura de influencias inglesas, con construcciones de ladrillo a la vista, perfiles salientes que configuran algunas pilastras y detalles en la cornisa o desarrollo de los edificios. Las cubiertas son de chapa y a dos aguas.

Los antiguos corrales y la báscula (construcción posterior al resto de las edificaciones ferroviarias) se encuentran en ruina. La nula utilidad ha llevado a que se deterioren con el tiempo y sufran desmantelamientos por parte de algunos residentes. El equipamiento que aún se puede ver en el sector lleva el mismo destino: señalética destruida por el paso y las inclemencias del tiempo, durmientes que han sido expoliados y tramos de rieles oxidados dan cuenta de una época de progreso en el poblado.

Se ha dejado para el final a los antiguos galpones de acopio, dado que han sido los disparadores de las iniciativas de gestión patrimonial surgidas en la localidad. Hasta hace algunos años se podían identificar un total de cinco galpones. La falta de concientización acerca del cuidado del patrimonio y el desconocimiento respecto a la historia local de algunos nuevos pobladores, adicionado a otros residentes de poblados vecinos, dio origen a un proceso de saqueo y desguace de estas edificaciones. Cabe aclarar que las construcciones en mejores condiciones eran utilizadas por algunos propietarios de campos aledaños para almacenar cereal, sin pago ni mantenimiento alguno, mientras que los restantes permanecian sin uso específico.

Las iniciativas de gestión patrimonial se iniciaron cuando uno de los galpones, el de mayores dimensiones, comenzó un proceso de deterioro notorio debido al hurto de chapas y tirantes (Figuras 5 y 6). Los vecinos más antiguos de Sundblad advirtieron la situación y adoptaron medidas urgentes que protegieran el legado y frenaran el saqueo: se redactó un Proyecto de Ordenanza para declarar Patrimonio Histórico y Urbano Municipal al complejo ferroviario de la localidad, iniciativa que se concretó luego de casi un año de tratativas con la generación de un instrumento legal de gestión que reconoce el valor que guarda ese conglomerado de bienes muebles e inmuebles; así, la Ordenanza Municipal № 3583/14 (Honorable Concejo Deliberante de Rivadavia, 2014), además de patrimonializar los recursos culturales, determinó la asignación de una obligación al ámbito público local. En el artículo 2 se manifiesta que se deberán "realizar los estudios necesarios que permitan diagnosticar la situación edilicia de los bienes y proponer alternativas de preservación patrimonial acorde a los usos actuales". Si bien a la fecha han pasado siete meses de su sanción, el municipio no ha realizado hasta el momento tarea alguna.

Ante la falta de compromiso y respuesta por parte del ámbito público, miembros de la Escuela Primaria № 12, única institución local en Sundblad, han propuesto diversas iniciativas en pos de la mejora de la calidad de vida de la comunidad, entre ellas la refuncionalización de uno de los galpones. El proyecto, denominado: "La recreación en el ámbito escolar como alternativa de revitalización patrimonial en la localidad de Sundblad", tiene como objetivo contribuir al desarrollo del esparcimiento y la recreación en el ámbito escolar, como uso social alternativo para la puesta en valor patrimonial. En este caso se pretende crear un espacio para el desarrollo de deportes y actividades recreativas para ser utilizado por los estudiantes y los pobladores en general, debido a que en el poblado no se cuenta con un predio (abierto o cerrado) destinado a tal función. Con tal iniciativa se pretende contribuir a la revitalización del sitio y, además, a la toma de conciencia por parte de los residentes de la importancia que revisten los lugares históricos como símbolo de la identidad comunitaria. Se debe destacar que el proyecto ha sido entregado y explicado por la directora de la institución escolar a miembros del gabinete de la Municipalidad de Rivadavia y actualmente se encuentra en evaluación.

\section{Actores y relaciones de poder a escala local}

Analizar las problemáticas en torno a la gestión del patrimonio cultural, implica conocer el mapa de actores y sus relaciones tanto en la localidad 


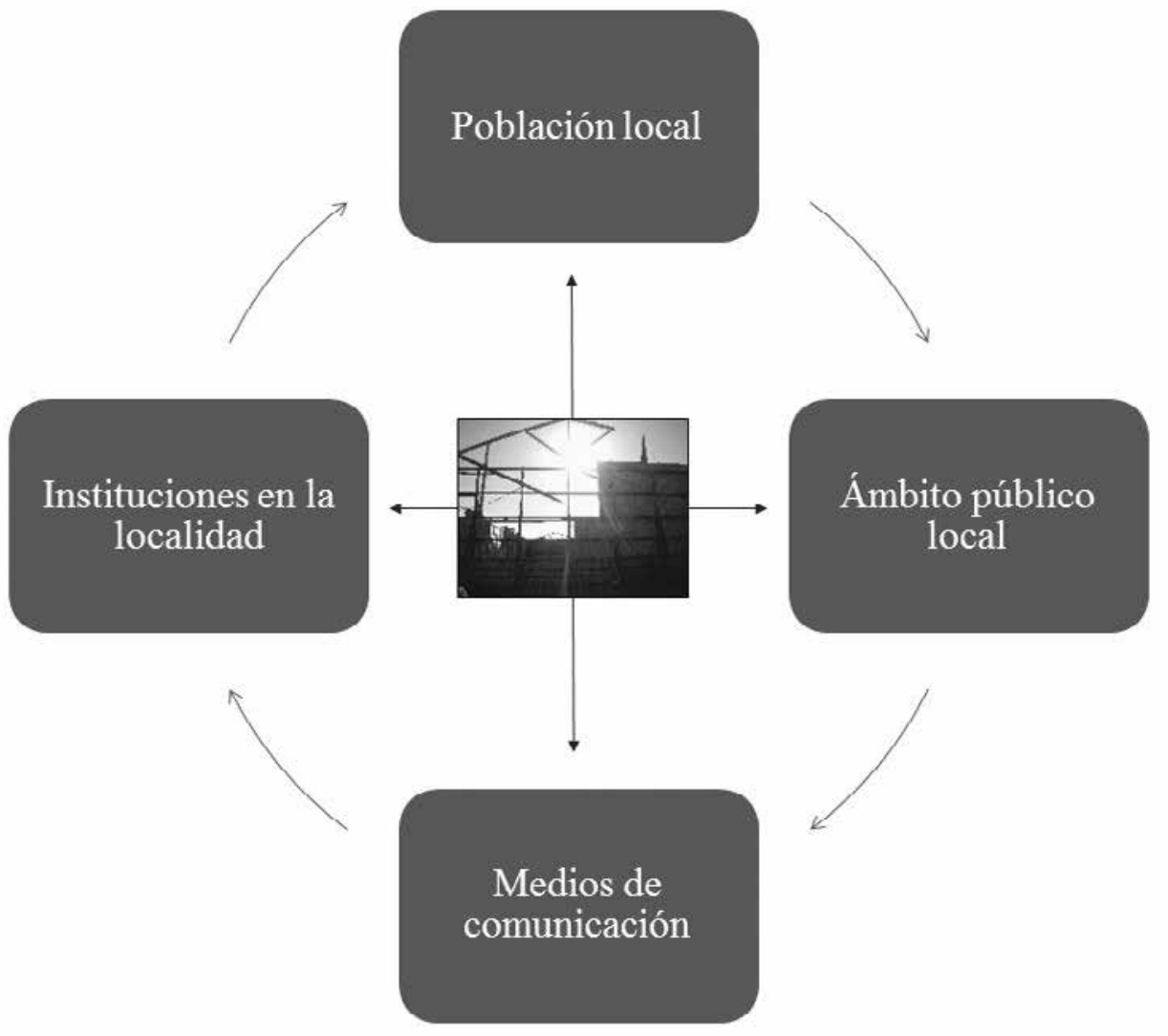

como en el ámbito del partido de Rivadavia (Figura 7).

En el centro de la escena están los residentes de Sundblad, como portadores del patrimonio local. Su responsabilidad radica en el deber de custodia y respeto hacia ese legado cultural. Ello implica evitar acciones vandálicas que atenten contra los bienes ferroviarios, al igual que sobre otras obras arquitectónicas del pueblo. Asimismo, la obligación de denuncia ante saqueos se configura como otra acción prioritaria. Ahora bien, ¿se llevan a cabo estas acciones a escala local? En este caso se debe realizar una distinción entre los antiguos y los nuevos pobladores. La diferencia dada por la configuración de un espacio vivido, arraigado y estructurado sobre una dimensión temporal de vivencia mayor en el pueblo, adicionado al arraigo y apreciación por lo local, posibilita distinguir entre aquellos residentes conscientes del valor colectivo que representa ese legado, y aquellos que visualizan en el patrimonio ocioso una oportunidad para obtener materiales (chapas y tirantes), de forma fácil y accesible. En este contexto se observa que los pobladores tradicionales guardan cierto respeto hacia el patrimonio local y registran una participación más activa para con el mismo, mientras que en
Figura 7:

Actores y vínculos a escala local

Fuente:

Elaboración propia (2014)

aquellos que hace pocos años residen en ella, la indiferencia hacia los valores históricos se traduce en acciones de desvalorización patrimonial que atentan contra la integridad física de los bienes.

Asociadas a los vecinos que residen en Sundblad, se debe hacer referencia a las instituciones locales. En el poblado se destaca la Escuela Primaria No 12 "Julio Argentino Roca", única organización que no solo funciona como ente promotor de la educación de los niños, sino que de ella surgen diferentes iniciativas que contribuyen a la mejora de la calidad de vida de la población. Entre los distintos proyectos se pueden destacar aquellos destinados a la recolección y separación de residuos en sus domicilios, la cría de aves, el desarrollo de huertas y, vinculada a la esfera patrimonial, la ya mencionada refuncionalización de uno de los antiguos galpones de acopio. Entre las dificultades que manifiesta la directora se destacan la carencia de recursos económicos para llevar a cabo cualquier actividad y la falta persistencia a la hora emprender y gestionar un proyecto concreto. Asimismo, es una tarea con ciertas dificultades aunar criterios e intereses entre la población, el ámbito público y cualquier otra institución interviniente en el 
proceso, ya que cada grupo reúne caracteres y formas de actuar disímiles.

Por otro lado, el ámbito público local, a partir de la sanción de la Ordenanza Municipal que declara al conjunto ferroviario como patrimonio histórico, adquiere responsabilidad en cuanto a la asignación de fondos y la búsqueda de asesoramiento en lo relativo a la realización de un diagnóstico de situación y la gestión para la puesta en valor del sector. En este contexto, tanto la institución educativa local como algunos vecinos han sostenido entrevistas con representantes del sector público y han obtenido un compromiso de llevar a cabo ciertas propuestas de revitalización. Asimismo, miembros de la Dirección de Medio Ambiente de la Municipalidad de Rivadavia se han acercado a la institución educativa para escuchar, en plenario abierto, determinadas inquietudes en la comunidad. Si bien se debe manifestar que existe una apertura e interés por las disímiles inquietudes de la población, aún no se han visualizado iniciativas concretas.

En materia patrimonial, en el poblado hasta la actualidad no se ha plasmado ninguna acción de gestión que tienda a la revitalización de los componentes del patrimonio cultural de Sundblad. El proceso de institucionalización legal del complejo ferroviario como patrimonio municipal se posiciona como la primera estrategia que pone en el centro de la palestra al patrimonio y sus problemáticas asociadas a escala local.

Por último, se hace mención de los medios de comunicación. En este caso, al ser una pequeña localidad, las repercusiones de aquello expuesto en diferentes medios radiales y de prensa escrita han dado resultados mayores, pues se ha suscitado en este último tiempo la publicación de diversos artículos periodísticos (Periódico digital Master News, 2014a y 2014b), algunos con posturas alentadoras de la situación de Sundblad, y otros con una visión más crítica acerca del estado inadecuado de los bienes ferroviarios y otros sectores del poblado.

Para finalizar, se debe destacar que por ser una localidad de reducidas dimensiones, el rol y las relaciones de poder de los actores se ven potenciados ya que cualquier situación que atente contra la comunidad, sea patrimonial o no, se ve reflejada de forma inmediata en la población. Por ello, aquellos actores clave, como son el ámbito público local y los residentes, deben trabajar de forma conjunta para poder definir políticas lo- cales en torno a la puesta en valor patrimonial. En este contexto se visualiza una oportunidad de acción para el primer sector, dado que existe una comunidad emprendedora, con fuerza conjunta de trabajo, que cuenta con los recursos humanos y asesoramiento necesario para llevar a cabo estrategias a escala local.

\section{Reflexiones finales}

Hoy día el patrimonio ferroviario se configura como un legado sociocultural producto de un proceso territorial y económico, que tuvo su origen en una política de expansión y avance de la frontera.

Numerosos poblados del país en la actualidad se caracterizan por paisajes estáticos, sin vida, debido al proceso de decadencia en el que han entrado a partir del cierre de los ramales ferroviarios, adicionado a otros devenires que se suscitaron en la esfera nacional. En este contexto se incluye la localidad de Sundblad, portadora de un complejo férreo de gran relevancia por sus aspectos históricos y arquitectónicos, pero carente de una valorización social, traducida en espacios abandonados y en deterioro. Más que dar respuestas, cabe plantearse algunas preguntas: ¿Puede hacerse referencia en estos casos a bienes patrimoniales o solamente podríamos referirnos a ellos como bienes producto de la cultura? ¿Dónde queda el reconocimiento y apreciación de la sociedad? ¿Cuál es la responsabilidad del Estado? ¿Y de los vecinos?

Algunas posibles respuestas a estos interrogantes podrian plantearse de forma articulada a partir de una disciplina compleja como es la gestión del patrimonio cultural, la cual implica aunar criterios e intereses, articular disímiles actores y consensuar entre relaciones de poder, administrar recursos (económicos, humanos, etc.) y lograr los benéficos mayores posibles para todas las partes intervinientes; y por encima de lo anterior, implica la voluntad de hacer y concretar.

En el estudio de caso se mencionaron algunas acciones concretas de gestión patrimonial que se han llevado a cabo, tendientes a la puesta en valor de los recursos pertenecientes al complejo del antiguo Ferrocarril Oeste. Asimismo, como un aporte de la presente investigación, se manifiestan aquí algunos mínimos lineamientos que articulen las propuestas que se están desarrollando en la actualidad. 
En primera instancia, y de acuerdo al cumplimiento de la Ordenanza Municipal No 3583/14, que declara como Patrimonio Histórico al conjunto ferroviario, se considera necesario un relevamiento de los bienes inmuebles que lo integran a escala local, a fin de conocer y determinar en mayor profundidad sus criterios de valoración, y de diagnosticar el estado de su situación edilicia en pos de realizar las acciones de intervención arquitectónica correspondientes.

Por otro lado, se considera propicia la puesta en valor de la antigua casa del "guarda hilos", actualmente inutilizada. En este caso, resultaría interesante la instalación de una biblioteca popular que desarrolle actividades culturales en la localidad, dado que Sundblad no cuenta con un espacio de tales características, que propicie el intercambio social y comunitario entre los ciudadanos.

Por otro lado, resulta prioritaria la instalación de señalética de referenciación patrimonial en cada uno de los bienes inmuebles dado que funcionaría como punto de información permanente y como recordatorio del valor simbólico y formal que cada espacio guarda. En este caso, las técnicas de interpretación patrimonial resultarán fundamentales cuando se diseñe la cartelería.

Desarrollar acciones de gestión patrimonial implica abordar los bienes materiales y trabajar sobre los sujetos promotores de la valorización del legado cultural. En ese sentido, la institución educativa local debería implementar un proyecto de concientización acerca de esta problemática local y la importancia que reviste la salvaguarda del patrimonio, destinado a los alumnos de educación primaria, pero con alcance a todos los pobladores.

Por último, y como responsabilidad del ámbito público, es necesario extender las políticas de preservación al resto de los bienes que configuran el patrimonio local. Además del complejo ferroviario, Sundblad cuenta con un importante conjunto de edificios que se estructuran bajo un movimiento historicista, con características sencillas, pero representativas, que deben ser conservados como símbolo de la historia de la localidad y la región.

A partir de estos mínimos lineamientos se pretende contribuir a la puesta en valor y reconocimiento del conjunto de bienes que forjaron la historia del poblado. Para su cumplimiento se requiere un trabajo conjunto entre los actores in- tervinientes en el proceso de gestión. Por un lado, el sector público con su responsabilidad de bregar por los intereses de los ciudadanos; por el otro, el compromiso de los residentes e instituciones de la localidad para participar en las actividades que se emprendan y en el deber moral de custodia y cuidado de los componentes que constituyen parte de la herencia comunitaria. En este sentido, la labor articulada e integral de las partes interesadas se presenta como el único escenario posible que puede contribuir a la mejora de la situación local al poner en valor recursos en pos de la satisfacción de las necesidades socioculturales de la comunidad y de la mejora de su calidad de vida.

\section{Bibliografía}

Acebo, E. y Schlüter, R. (2012). Diccionario de turismo. Buenos Aires: Claridad.

Arias, N. (2012). Nuestro patrimonio cultural: una construcción colectiva. Clarín, Arquitectura. Patrimonio Argentino. Teatros, cines y auditorios (pp. 11-15). Buenos Aires: Arte Gráfico Editorial Argentino.

Ballart, J. y Tresserras, J. (2007). Gestión del patrimonio cultural ( $3^{\mathrm{a}}$ ed.). Barcelona: Ariel.

Camarero, C. y Garrido, M. (2008). Marketing del patrimonio cultural. Madrid: Pirámides.

CITAB (Centro de Investigaciones Territoriales y Ambientales Bonaerenses) (2004a). Historia y población. Buenos Aires: Banco Provincia. Recuperado de http://www.bpba.com. ar/citab/publicaciones/rivadavia/archivos/ histypoba.pdf.

CITAB (Centro de Investigaciones Territoriales y Ambientales Bonaerenses) (2004b). Reseña histórica de las escuelas. Buenos Aires: Banco Provincia. Recuperado de http://www. bancoprovincia.com.ar/citab/publicaciones/ rivadavia/indice.htm.

Conti, A. (2010). Nuevas categorías patrimoniales: del monumento histórico al territorio. En F. París y A. Novacovsky (comp.). Textos de cátedra IV (pp. 127-139). Mar del Plata: Universidad Nacional de Mar del Plata.

Costa, F. (2009). Turismo e património cultural: interpretação e qualificação. San Pablo: Editora Senac San Pablo. Ediciones SESC SP.

Crespi, M. y Planells, M. (2003). Patrimonio cultural. Madrid: Sintesis.

Feria, J. (2010). Patrimonio territorial y desarrollo sostenible: un estudio comparativo en lbe- 
roamérica y España. Estudios Geográficos, 268(LXXI), 129-159.

Feria, J. (2013). El patrimonio territorial: algunas aportaciones para su entendimiento y puesta en valor. Revista Electrónica de Patrimonio Histórico, 12, 1-25.

Ferrari, M. (2010a). Los asentamientos urbanos producidos por la instalación del ferrocarril en el noroeste argentino. Apuntes, 24(1), 26-43.

Ferrari, M. (2010b). El sistema ferroviario en el noroeste argentino. Arquitectura e instalaciones complementarias. Apuntes, 24(1), 44-61.

García, J. y De Medeiros, L. (2014). Storytelling Patrimonial. Curso Nuevas estrategias para Salvar el patrimonio (Proyecto de Innovación Docente). Universidad de Valladolid: España.

Gutiérrez, R. (2014). Repensando el patrimonio desde América Latina. En Zingoni, J. y Pinassi, A. (comp.). Gestión del patrimonio urbano. Textos de cátedra (1) (pp. 63-80). Bahía Blanca: Ediuns.

Hernández, F. (2002). El patrimonio cultural: la memoria recuperada. Asturias: Trea.

Honorable Concejo Deliberante de Rivadavia (2014). Ordenanza Municipal No 3583/14. América (Argentina).

lcomos (Consejo Internacional de Monumentos y Sitios) (1999). Carta internacional sobre turismo cultural: la gestión del turismo en los sitios con patrimonio significativo. México.

Instituto Nacional de Estadística y Censos (2010). Censo nacional de población, hogares y viviendas 2010. Recuperado de http://www.indec. gov.ar/nivel4_default.asp?id_tema_1=2\&tid_ tema_2=41Ctid_tema_3=135.

Mejía, M. y Valladares, P. (2011). Manual de gestión cultural para voluntarios. Tegucigalpa: Programa de Voluntarios de las Naciones Unidas (ONU).

Moreira-Wachtel, S. y Tréllez, E. (2013). La interpretación del patrimonio natural y cultural. Una visión intercultural y participativa. Lima: Ministerio de Ambiente del Perú.

Municipalidad de Rivadavia (2014). Historia de Sundblad. Recuperado de http://www.rivadavia.mun.gba.gov.ar/sundblad.htm.

Ortega, J. (1998). Patrimonio territorial: el territorio como recursos cultural y económico. Ciudades, 4, 33-48.

Pardo, C. (2008). Turismo y patrimonio industrial. Madrid: Síntesis.
Periódico Digital Master News (2014a, agosto 27). El Frente Renovador pide por el abandono que sufre Sundblad. Recuperado de http://www. masternews.com.ar/index.php?option=com content\&view=articlectid=34805:el-frenterenovador-pide-por-el-qabandonoq-queqsufreq-sundbladetcatid=19:la-ciudadctle$\operatorname{mid}=67$.

Periódico Digital Master News (2014b, agosto 28). Gisela Medeiro: Felicito a los vecinos de Sundblad por el compromiso con su comunidad. Recuperado de http://www.masternews. com.ar/index.php?option=com_contentetvi ew=articlectid=34824: gisela-medeiro-felicito-a-los-vecinos-de-sundblad-por-el-compromiso-con-su-comunidadetcatid=42:eldistritoctltemid $=90$.

Pinassi, A. (2012). La arquitectura italianizante como patrimonio cultural regional y potencial atractivo turístico recreativo. Caso de análisis: localidad de Sundblad (provincia de Buenos Aires, Argentina). Actas V Congreso Latinoamericano de Investigación Turística. San Pablo.

Prats, L. (1997). Antropología y patrimonio. Barcelona: Ariel.

Prats, L. (1998). El concepto de patrimonio cultural. Política y Sociedad, 27, 63-76.

Prats, L. (2005). Concepto y gestión del patrimonio local. Cuadernos de Antropología Social, $21,17-35$.

Prats, L. (2006). La mercantilización del patrimonio: entre la economía turística y las representaciones identitarias. $\mathrm{PH}$, Boletín del Instituto Andaluz del Patrimonio Histórico, 58, 72-80.

Prats, L. (2014). El carácter magmàtic del patrimoni etnològic. Etnologia. Revista D'Etnologia de Catalunya, 39, 152-159.

Querol, M. (2010). Manual de gestión del patrimonio cultural. Madrid: Akal.

Ramírez, 1. (1997). Memorias e historia de Sundblad (manuscrito no publicado). Juan José Paso (Argentina).

Rodrigues Da Silva, R. (2010). El patrimonio industrial: posibilidades para el paisaje cultural. Actas del $X$ Congreso Internacional CICOP. Santiago de Chile.

Schvarzer, J. (1999). Los ferrocarriles de carga en la Argentina. Problemas y desafíos en vísperas del siglo XXI. Documento de traba- 
jo $N^{\circ}$ 2. Buenos Aires: Facultad de Ciencias Económicas, Universidad de Buenos Aires.

Tartarini, J. (2005). Arquitectura ferroviaria. Buenos Aires: Colihue.

TICCIH (Comité Internacional para la Conservación del Patrimonio Industrial) (2003). Carta de Nizhny Tagil sobre el patrimonio industrial. Moscú (Rusia).

Torrejón, A. (2013). Tesauro turístico argentino. Buenos Aires: Ministerio de Turismo de la Nación.

Torres, M. (2008). Caminos, carretas, vías, ferrocarriles y pueblo. En Torres, M. (Ed.).
Historias ferroviarias al sur del Salado (pp. 19-46). Mar del Plata: EUDEM.

Velasco, M. (2009). Gestión turística del patrimonio cultural: enfoques para un desarrollo sostenible del turismo cultural. Cuadernos de Turismo, 23, 237-253.

Zingoni, J. (2014). Pensar el patrimonio arquitectónico. En Zingoni, J. y Pinassi, A. (comp.). Gestión del Patrimonio Urbano. Textos de cátedra (1) (pp. 15-34). Bahía Blanca: Ediuns.

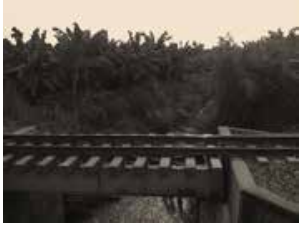

